\title{
Nonlinear loss in $\mathrm{Nd}$-doped laser glass
}

\author{
A. Penzkofer and W. Kaiser \\ Physik-Department der Technischen Universtät München, Munich, Germany \\ (Received 31 July 1972) \\ The amplifier gain and the energy transmission in Nd-glass were investigated as functions \\ of light intensity using single picosecond pulses. The observed reductions of gain and trans- \\ mission are well accounted for by a two-photon absorption process in the $\mathrm{Nd}^{3+}$ system. \\ There is good agreement between the measured and calculated two-photon absorption cross \\ section of $\sigma^{\prime}=(1.3 \pm 0.3) \times 10^{-32} \mathrm{~cm}^{4} / \mathrm{W}$. The two-photon absorption limits the obtainable out- \\ put intensity in a Nd-glass amplifier and reduces the self-focusing action in the material.
}

During the past years Nd-doped glass has received increasing attention in the construction of high-power laser systems: Several authors have noticed decreasing gain for Nd-glass at high power or at a high energy density for short input pulses. ${ }^{1-3}$ Two explanations have been offered for this effect: self-focusing for single ultrashort light pulses, ${ }^{2}$ and filling of the lower laser level for trains and groups of picosecond pulses. . $^{3}$

In our work we used single intense light pulses of 6-psec duration for the study of the nonlinear gain and the nonlinear loss of pumped and unpumped Nd-glass. The peak intensity of the laser pulses was monitored by a system using a saturable dye. ${ }^{4}$ Pulse energies were measured with fast phototubes and oscilloscopes (time constant, $\simeq 0.5 \mathrm{nsec}$ ).

In Fig. 1(a) we present the energy transmission at $\lambda$ $=1.06 \mu$ of a $32-\mathrm{cm}$-long glass rod doped with $3 \%$
$\mathrm{Nd}_{2} \mathrm{O}_{3}$ (Schott LG 630). At an input intensity of $I_{0}=2$ $\times 10^{10} \mathrm{~W} / \mathrm{cm}^{2}$, the transmission is reduced to $60 \%$, indicating the nonlinear loss of the material. The same rod was investigated in a conventional amplifier configuration (two passes). The experimental points in Fig. 1(b). show quite clearly the decreasing energy gain, from 320 to 210 , for increasing input intensity $(1.06 \mu)$. The energy transmission of a glass rod (length, $14.7 \mathrm{~cm}$ ) which contained $3 \% \mathrm{La}_{2} \mathrm{O}_{3}$ instead of $\mathrm{Nd}_{2} \mathrm{O}_{3}$ is depicted in Fig. 1 (c). This material does not show the nonlinear loss found in the $\mathrm{Nd}$-doped specimen. It is interesting to see that the residual absorption (at $1.06 \mu$ ), mainly due to $\mathrm{Fe}^{2+}$ impurities, ${ }^{506}$ is bleached at very high input intensities. The energy transmission of a glass sample doped with $5 \% \mathrm{Nd}_{2} \mathrm{O}_{3}$ (Schott LG 650) showed a larger nonlinear loss. Measurements of beam diameter and beam divergence in front of and behind the various glass rods indicate a slight increase of the beam diameter and a 


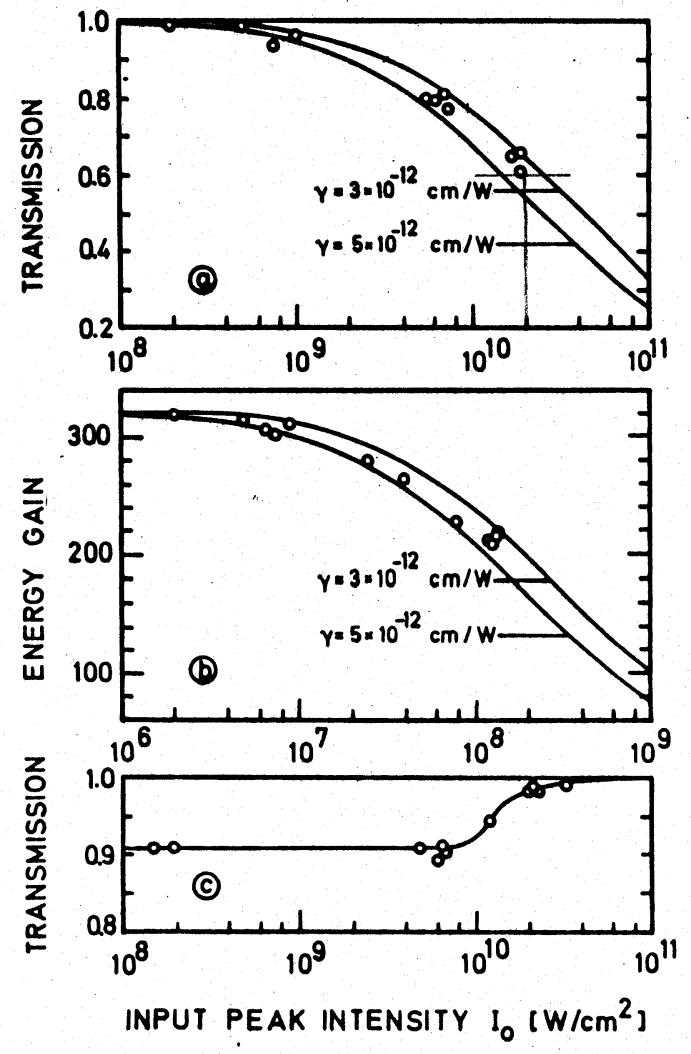

FIG. 1. Amplifier gain and energy transmission versus input peak intensity. (a) Energy transmission of a Schott LG 630 sample of $32-\mathrm{cm}$ length. (b) Amplifier gain of the same rod (two passes). (c) Energy transmission of a glass sample with $3 \% \mathrm{La}_{2} \mathrm{O}_{3}$. The curves in (a) and (b) are calculated assuming an intensity-dependent absorption coefficient $\gamma I$.

very small decrease of the beam divergence. At $80{ }^{\circ} \mathrm{K}$, the same nonlinear loss was measured in a Nd-glass sample (Schott LG 630) as is found at room temperature.

Before discussing the intensity-dependent absorption and its origin, the following points should be mentioned.

(i) Gain saturation due to depletion of the upper laser level ${ }^{7}$ or filling of the terminal laser level ${ }^{3}$ is ruled out in our experiments with single picosecond pulses. We have selected two succeeding pulses of the pulse train (time interval, $10 \mathrm{nsec}$ ) and measured the gain at $I_{0}$ $=2 \times 10^{8} \mathrm{~W} / \mathrm{cm}^{2}$. Both pulses experienced the same amplification. This observation is supported by the following numbers: From our small-signal gain we calculate a gain coefficient of $\beta=0.1 \mathrm{~cm}^{-1}$, which suggests an inversion of $N=5 \times 10^{18} \mathrm{~cm}^{-3}\left(\sigma=2.1 \times 10^{-20} \mathrm{~cm}^{2}{ }^{8}\right)$ or an energy density stored within the material of $0.9 \mathrm{~J} / \mathrm{cm}^{3}$. For $I=2 \times 10^{10} \mathrm{~W} / \mathrm{cm}^{2}$ and a pulse duration of $t_{p}=6 \mathrm{psec}$, the energy density removed is estimated to be $I \beta t_{p}$ $=0.012 \mathrm{~J} / \mathrm{cm}^{3}$; i.e., the upper laser level is not changed significantly and the terminal laser level is not filled appreciably. (ii) Stimulated Raman scattering appears to be unimportant in our investigations. At $I=2$ $\times 10^{10} \mathrm{~W} / \mathrm{cm}^{2}$, for a sample length of $30 \mathrm{~cm}$ and a Raman gain factor (at $1.06 \mu$ ) of $g_{R}=2 \times 10^{-11} \mathrm{~cm} / \mathrm{W}, 9$ we calculate a Raman gain of $e^{12}$, which results in a conversion of about $10^{-7}$. (iii) We detected, as expected, no stimulated backward scattering in the amplifier even at the highest input intensities. (iv) Self-focusing (due to the nonlinear refractive index $n_{2}$ ) did not occur in our experiments, as shown by measurements of the beam diameter and the beam divergence. The interaction of $n_{2}$ with the intensity-dependent absorption will be discussed below.

Introducing a loss, $\gamma I$, proportional to the intensity $I$, we write.

$$
\frac{d I}{d x}=(\beta-\alpha) I-\gamma I^{2},
$$

which gives the solution

$$
I_{\text {out }}=I_{\text {in }} \frac{\exp (\beta-\alpha) l}{1+\gamma I_{\text {in }}[\exp (\beta-\alpha) l-1] /(\beta-\alpha)} .
$$

$\beta$ is the gain factor of the Nd-glass sample, $\alpha$ is the linear absorption coefficient of the material, and $l$ is the length of the sample. Equation (2) shows quite clearly the reduced gain due to the nonlinear absorption. In fact, there is a maximum value of output intensity, $I_{\max }$ $=(\beta-\alpha) / \gamma$, for long sample length $l$ (see aloo Ref. 10). Integrating Eq. (2) over space and time, we calculated the energy transmission and the energy gain for various values of $\gamma$. The two curves drawn in Figs. 1(a) and $1(\mathrm{~b})$ suggest a value of $\gamma=(4 \pm 1) \times 10^{-12} \mathrm{~cm} / \mathrm{W}$ for glass of $3 \%$ $\mathrm{Nd}_{2} \mathrm{O}_{3}$ content. It should be emphasized that the same value of $\gamma$ is found for pumped and unpumped Nd-glass and at 300 and $80^{\circ} \mathrm{K}$. Glass specimens with $5 \% \mathrm{Nd}_{2} \mathrm{O}_{3}$ concentration gave a higher value of $\gamma=(7 \pm 2) \times 10^{-12} \mathrm{~cm} / \mathrm{W}$, while the undoped glass in Fig. 1(c) does not show any nonlinear loss. The intensity-dependent loss appears to be proportional to the concentration of $\mathrm{Nd}^{3+}$ ions in the glass matrix.

As to the physical origin of the intensity-dependent loss, two processes - two-photon absorption and two-step absorption - have to be considered. The cross section $\sigma^{(2)}$ for a two-photon transition ${ }^{11}$ is given by

$$
\sigma^{(2)}=\sigma^{\prime} I=\frac{\pi \omega_{L} g\left(2 \omega_{L}-\omega_{b a}\right)}{4 \hbar^{3} I}\left|\sum_{a} \frac{\left\langle b\left|H^{\prime}\right| q\right\rangle\left\langle q\left|H^{\prime}\right| a\right\rangle}{\omega_{L}-\omega_{a a}}\right|^{2},
$$

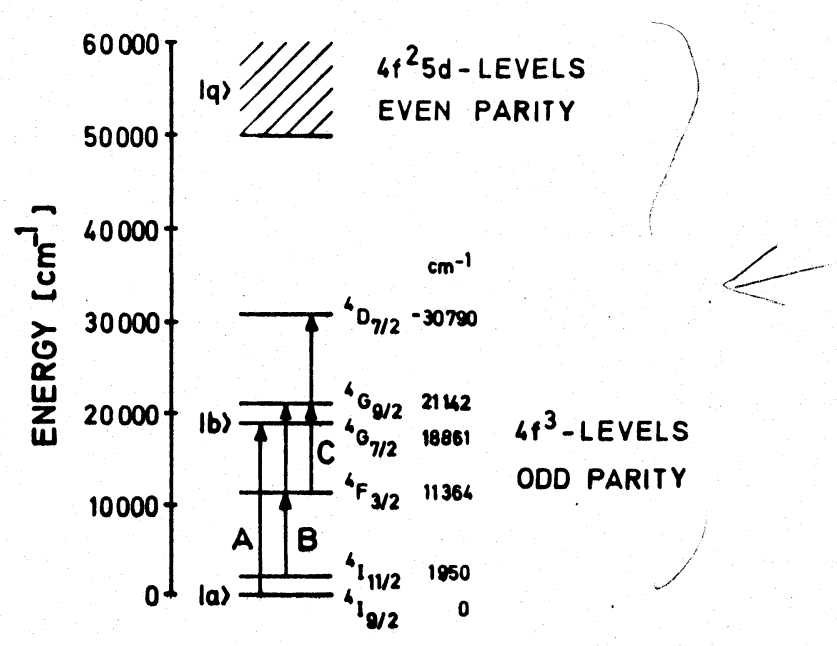

FIG. 2. Two-photon and two-step absorption processes in Ndglass. A, two-photon absorption from the ground state; B and $C$, two-step absorption from the terminal laser level and from the upper laser level, respectively. 


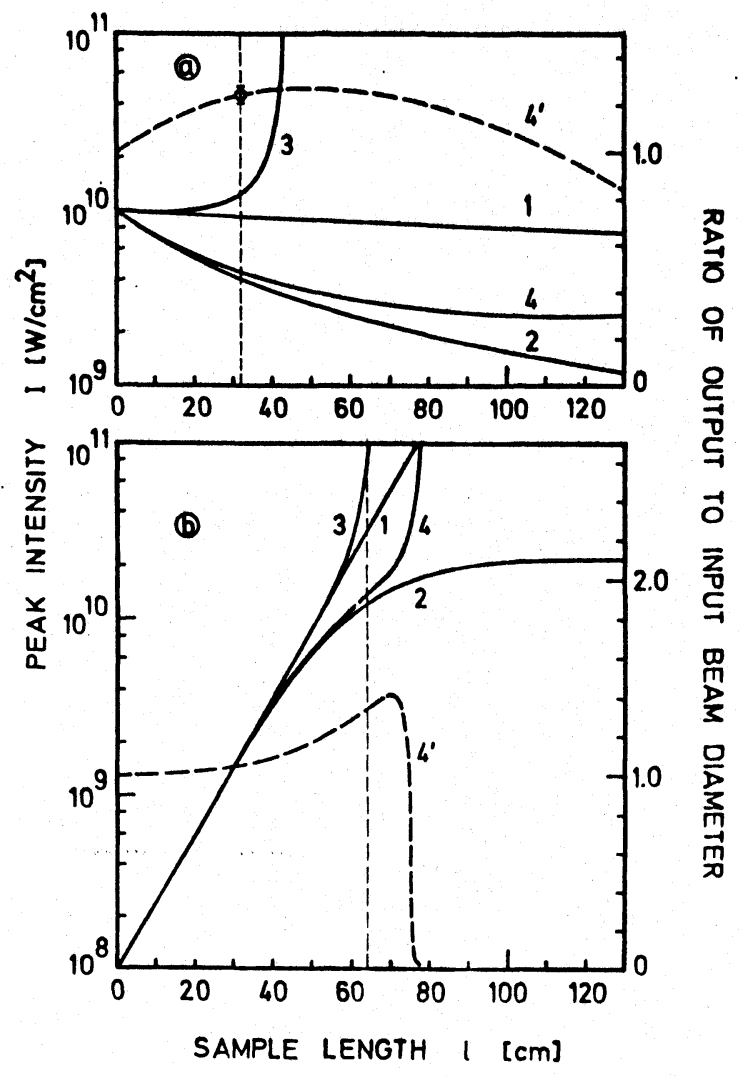

FIG. 3. Peak intensity and beam diameter in Nd-glass (Schott LG 630) as a function of sample length: (1) no nonlinear processes; (2) with two-photon absorption $\left(\gamma=4 \times 10^{-12} \mathrm{~cm} / \mathrm{W}\right)$; (3) with nonlinear refractive index $\left(n_{2}=2.2 \times 10^{-13} \mathrm{esw}\right.$; (4) and $\left(4^{\prime}\right)$ with two-photon absorption and nonl inear refractive index. Full lines, output peak intensity; broken lines, ratio of output to Input diameter; vertical lines, effective rod length. (a) Sample not excited; (b) sample pumped.

where $\omega_{L}$ is the laser frequency, $g\left(2 \omega_{L}-\omega_{b a}\right)$ is the line shape of the transition, $\omega_{b a}=\omega_{b}-\omega_{a}$ is the frequency separation between the upper state $|b\rangle$ and the lower state $|a\rangle$ of the two-photon transition, $I$ is the laser intansity, $q$ is the counting index for the levels $|q\rangle$ that are separated from the lower state $|a\rangle$ by $\omega_{a a}=\omega_{a}-\omega_{a}$, and $H^{\prime}$ is the interaction Hamiltonian. The single-photon matrix elements $\left\langle b\left|H^{\prime}\right| q\right\rangle$ and $\left\langle q\left|H^{\prime}\right| a\right\rangle$ are large for allowed electric dipole transitions. The product of these matrix elements involves the following selection rules for strong two-photon absorption: $\Delta J=0, \pm 1, \pm 2 ; \Delta L=0, \pm 1, \pm 2 ; \Delta S$ $=0$; and no change in parity. In addition, the state $|a\rangle$. must be occupied, and one requires $\omega_{b a} \sim 2 \omega_{L}$. These conditions are fulfilled ${ }^{12}$ for transition A (Fig. 2). Remembering sum rules ${ }^{11}$ for the operator $\Sigma_{q}|q\rangle\langle q|$ and as suming a Gaussian line shape for the transition, we rewrite Eq. (3) as

$\sigma^{\prime}=\frac{(4 \pi \ln 2)^{1 / 2} \omega_{L} e^{4}\left|\left\langle a\left|r^{2}\right| b\right\rangle\right|^{2}}{9 \Delta \omega_{G} \eta^{2} c^{2} \epsilon_{0}^{2} \hbar^{3}\left(\omega_{L}-\bar{\omega}\right)^{2}} \exp \left[-4 \ln 2\left(\frac{2 \omega_{L}-\omega_{b a}}{\Delta \omega_{G}}\right)^{2}\right]$

$\bar{\omega}=\omega_{a}-\omega_{a}$ is an average of the different frequency separations $\omega_{a a}$. The following data are relevant for our glass sample (Schott LG 630): $\omega_{L}=1.785 \times 10^{13} \mathrm{sec}^{-1}, \eta$ $=1.509, \Delta \omega_{G}=4.7 \times 10^{13} \mathrm{sec}^{-1}, \omega_{b Q}=3.555 \times 10^{15} \mathrm{sec}^{-1}$, $\left|\left\langle a\left|r^{2}\right| b\right\rangle\right|=0.39 \times 10^{-16} \mathrm{~cm}^{2},{ }^{13}$ and $\bar{\omega}=9.4 \times 10^{16} \mathrm{sec}^{-1}$ $\left(56000 \mathrm{~cm}^{-1}\right) .12$ With these numbers we calculate $\sigma^{\prime}=1.4$ $\times 10^{-32} \mathrm{~cm}^{4} / \mathrm{W}$. This value is in good agreement with the measured number of $\sigma^{\prime}=\gamma / N_{\mathrm{Nd}^{3+}}=(1.3 \pm 0.3) \times 10^{-32} \mathrm{~cm}^{4} /$ $\mathrm{W}\left(N_{\mathrm{Nd}^{\mathrm{s}}+}=3.2 \times 10^{20} \mathrm{~cm}^{-9}\right)$.

The two-step absorption coefficient is approximately given by $\gamma=\sigma_{1} \tau \sigma_{2} N / \hbar \omega_{L}$, where $\sigma_{1}$ and $\sigma_{2}$ are the absorption cross sections for single-photon absorption, $\tau$ is the lifetime of the intermediate state, and $N$ is the density of the starting level. Two-step absorption is possible for transitions B and C (Fig. 2). For transition B we estimate $\gamma=1.5 \times 10^{-13} \mathrm{~cm} / \mathrm{W}$ at $300^{\circ} \mathrm{K}$, and $\gamma=10^{-24} \mathrm{~cm} /$ W at $80^{\circ} \mathrm{K} .\left[\sigma_{1} \propto \sigma_{2} \approx 10^{-8}\langle r\rangle^{2} \approx 4 \times 10^{-23} \mathrm{~cm}^{2},{ }^{12,13} \tau\left({ }^{4} F_{3 / 2}\right)\right.$ $=6.4 \times 10^{-4} \mathrm{sec}, N \approx 2.4 \times 10^{16} \mathrm{~cm}^{-3}$ at $300^{\circ} \mathrm{K}$, and $N \approx 2$ $\times 10^{5}$ at $80^{\circ} \mathrm{K}$.] For transition $\mathrm{C}$ (starting from the upper laser level) we calculate $\gamma=4 \times 10^{-10} \mathrm{~cm} / \mathrm{W}\left(\sigma_{1} \simeq \sigma_{2} \simeq 4\right.$ $\times 10^{-23} \mathrm{~cm}^{2}, \tau\left({ }^{4} G_{9 / 2}\right) \simeq 10^{-8} \mathrm{sec}, N \approx 5 \times 10^{18} \mathrm{~cm}^{-3}$, pumped rod). The values for $\gamma$ estimated here are substantially smaller than the experimentally determined loss coefficient. Our measurements in pumped and unpumped rods and our data at $80^{\circ} \mathrm{K}$ rule out the two-step process.

Now we turn to investigations which include the intensity-dependent refractive index $n=n_{0}+n_{2} I$. We computed the change of a Gaussian input pulse with peak intensity $I_{0}$, beam diameter $a$ ( $1 / e$ value), and divergence $\Theta$ (outside the rod) when passing through a pumped and unpumped Nd-glass rod. ${ }^{14}$ Four cases were considered for comparison: (1) Nonlinear processes are not present; (2) an intensity-dependent loss with $\gamma=4 \times 10^{-12} \mathrm{~cm} /$ $W$ was included; (3) the nonlinear refractive index $n_{2}$ $=2.2 \times 10^{-13} \mathrm{esu}^{15,16}-1$.e., . self-focusing was considered $^{14}$; and finally (4) and (4') the effect of both nonlinearities, $\gamma I$ and $n_{2} I$, was calculated.$^{17}$ In Fig. $3(a)$ the change of the peak intensity and of the beam diameter is shown as a function of the length of the Nd-glass rod (unpumped, $\left.I_{0}=10^{10} \mathrm{~W} / \mathrm{cm}^{2}, a=4.4 \mathrm{~mm}, \Theta=3.4 \times 10^{-4}\right)$. (1) Without nonlinear effects the intensity decreases linearly on account of the beam divergence. (2) The loss $\gamma I$ reduces the peak intensity strongly. (3) The nonlinear refractive index leads to self-focusing at a sample length of $40 \mathrm{~cm}$ : (4) The intensity-dependent loss reduces the self-focusing action. For short samples the peak intensity is similar to case (2). (4') Of interest is the increasing beam diameter $(l<50 \mathrm{~cm})$ followed by a slow decrease. The experimentally measured point agrees well with the calculated curve. In Fig. 3(b). the peak intensity and the beam diameter are calculated for a Nd-glass amplifier $\left(\beta=0.093 \mathrm{~cm}^{-1}, I_{0}=10^{8} \mathrm{~W} / \mathrm{cm}^{2}, a\right.$ $\left.=3.8 \mathrm{~mm}, \theta=4 \times 10^{-4}\right)$. (1) Without nonlinearities the intensity rises linearly. (2) Nonlinear absorption leads to a maximum intensity, $I_{\text {max }}$, which has been discussed in connection with Eq. (2). The value $I_{\max }=(\beta-\alpha) / \gamma$ $=2.25 \times 10^{10} \mathrm{~W} / \mathrm{cm}^{2}$ is approximately reached for a distance of $90 \mathrm{~cm}$. (3) The nonlinear refractive index leads to self-focusing at a sample length of $\sim 65 \mathrm{~cm}$. (4) Nonlinear absorption reduces the self-focusing action to a self-focusing length of $\sim 80 \mathrm{~cm}$. (4') First, the beam diameter increases due to the intensity-dependent loss; later, rather abruptly self-focusing sets in.

In summary, we can say that the nonlinear absorption affects the Nd-glass amplifier in the following ways: 
The over-all gain is reduced and the beam diameter as well as the self-focusing length are increased. The latter two effects occur since the intensity-dependent loss reduces preferentially the higher light intensity in the center of the beam.

The authors thank Dr. A. Laubereau and Dr. M. Maier for valuable discussions, and Dr. N. Neuroth for supplying the $3 \% \mathrm{La}_{2} \mathrm{O}_{3}$ glass rod used in their experiment. The authors are also grateful to Dr. M. Maier and $O$. Rahn for the computer program used for the calculation of the self-focusing action in their glass samples.

${ }^{1}$ C. R. Jones, P.V. Avizonis, and P. Sivgals, Natl. Bur. Std. Special Publication No. 341 (U.S. GPO, Washington, D.C., 1970), p. 28.

${ }^{2}$ N. G. Basov, I. Kertes, P.G. Kryukov, Yu. A. Matveets, Yu.V. Senatskii, and S.V. Chekalin, Sov. Phys. JETP 33, 289 (1971).

${ }^{3}$ P. C. Magnante, IEEE J. Quantum Electron. QE-8, 440 (1972).

${ }^{4}$ A. Penzkofer, D. von der Linde, and A. Laubereau, Opt. Commun. 4, 377 (1972).

${ }^{5}$ C. R. Bamford and E.J. Hudson, Technical Papers of the 7th Intermational Congress of Glass (Gordon and Breach, New York, 1966), Chap. 6.
${ }^{6}$ N. Neuroth, R. Hasse, and A. Knecht, Natl. Bur. Std Special Publication No. 356 (U.S. GPO, Washington, D. C. , 1971), p. 3.

${ }^{7}$ A. Y. Cabezas, G.L. Allister, and W.K. Ng, J. Appl. Phys. 38,3487 (1967).

${ }^{8}$ M. Birnbaum and J. A. Gelbwachs, J. Appl. Phys. 43, 2335 (1972).

${ }^{9}$ R. H. Stolen, E. P. Ippen, and A.R. Tynes, Appl. Phys. Letters 20, 62 (1972).

${ }^{10}$ F. V. Bunkin and A. M. Prokhorov, Sov. Phys. JETP 21, 725 (1965).

${ }^{11}$ R. H. Pantell and H.E. Puthoff, Fundamentals of Quantum Electronics (Wiley, New York, 1969).

${ }^{12}$ G. H. Dieke, Spectra and Energy Levels of Rare Earth Ions in Crystals, edited by H.M. Crosswhite and H. Crosswhite (Wiley, New York, 1968).

${ }^{13}$ B. R. Judd, Phys. Rev. 127, 750 (1962).

${ }^{14} \mathrm{~A}$ computer program of M. Maier and O. Rahn was used here. See M. Maier, O. Ralin, and G. Wendel, Z. Naturforsch. 25a, 1868 (1970); O. Kihn and M. Maier, Phys. Rev. Letters 29, 558 (1972).

${ }^{15} \mathrm{~J}$. Davit, Natl. Bur. Std. Specilal Publication No. 341 (U.S. GPO, Washington, D. C., 1970), p. 37.

${ }^{16}$ M. A. Duguay, J.W. Hiansen, and S. L. Shapiro XIEEE J. Quantum Electron. QE-6, 725 (1970).

${ }^{17}$ The calculations of Ref. 14 were expanded to include $\gamma I$. [See also A. L. Dyshko, V.N. Lugovol, and A.M. Prokhorov, Zh. Eksperim. i Teor, Fiz. 61, 2306 (1971).] 